the genesis of macromosaic structure, probably subsequent to the twin formation-analogous to the behaviour of copper upon solidifying. It is believed that the forms assumed by the various constituents in steel, in particular the so-called Widmanstätten figures observed in alpha iron after quenching from above the critical range, are the result of the twinning and macromosaic lattice movements rather than of the crystallographic uniformity of the gamma-toalpha transformation process, as was proposed by Mehl and Smith.

Results will be published at an early date. Alden B. Greninger.

Metallurgical Laboratories, Harvard University.

\section{Pyrites in Quartz}

Pyrites $\left(\mathrm{FeS}_{2}\right)$ in the form of small crystals and specks of a brassy-yellow colour may be found frequently in quartz. Four pieces of Brazilian quartz, containing pyrites inclusions, have now been found which are of unusual interest.

One of these pieces is a well-shaped cap of good optical quality, very clear and completely devoid of feathers, 'smoke', etc., and containing three large pyrites crystals and a number of smaller ones. Their colour is almost a pure white and they have the characteristic pentagonal dodecahedron form and striated faces. It is very rarely that such fine inclusions are found in so clear a piece of quartz. The specimen is interesting also in that it has two beautifully etched faces.

A fifth specimen has also been found having a complete hole (approx. $10 \times 4 \times 8 \mathrm{~mm}$.), in which a crystal doubtless at one time existed, for the faces of the hole are striated corresponding to the structure of the pyrites crystal. Unfortunately, in the process of working the quartz the hole was cut into, so that its vapour content escaped before an examination could be made.

A superficial examination indicates that the inclusions tend to lie in one particular plane through the quartz, and, further, the water inclusions in one of the specimens also lie in the same plane.

A fuller examination of the specimens is now in progress.

F. BRECH.

\section{Research Laboratory, \\ Adam Hilger, Ltd., \\ 98 Kings Road, N.W.1. May 3.}

\section{The Minimum in the Gamma Function}

Ir is well known that the gamma function $\Gamma(z)$ for real and positive values of $z$ has a minimum between $z=1 \cdot 46$ and $1 \cdot 47$. In a number of texts on the theory of functions it is stated that the minimum occurs at $z=1 \cdot 4616321 \ldots$ and that the corresponding value of $\Gamma(z)$ is $0.8856032 \ldots$ Only one text that we have examined, namely Joseph Edwards's monumental work "Integral Calculus", vol, 2, chap. xxiv (Macmillan, 1922), gives any indication of how the minimum points can be calculated. The method therein explained depends on certain properties of the gamma function and of the related logarithmic derivative $\mathrm{d} \ln \Gamma(z+1) / d z$, commonly written $\psi(z)$, following Gauss. At best, the problem is finally one in successive approximation.

There is another approach to the problem, admittedly crude, but effective. Gauss ("Werke", 3,
161-162) left us a table of $\log \Gamma(z+1)$ to twenty decimals, and a table of its logarithmic derivative, $\psi(z)$, to eighteen decimals, by steps of 0.01 in the argument $z$ between $z=0$ and 1 . It should be possible, then, by inverse interpolation in Gauss's table to find the value of $z$ for which $\psi(z)$ vanishes. This value of $z$ will be the abscissa of the minimum in $\Gamma(z+1)$, so that again by interpolation it should be possible to find the minimum value of $\log \Gamma(z+1)$. Finally, by Thompson's twenty place logarithms ("Tracts for Computers", No. 14, Cambridge, 1927) the minimum in $\Gamma(z)$ jtself can be determined.

Using Everett's interpolation formula, and retain. ing sixth differences, we have in this manner found that the zero of $\psi(z)$, and hence the minimum in log $\Gamma(z)$ and in $\Gamma(z)$ occurs at $z=1 \cdot 461632144968362268$. Interpolation with the same formula gives the minimum of $\log \Gamma(z)$ equal to $9 \cdot 94723917439338526292$; whence, by Thompson's logarithms, it turns out that the minimum of $\Gamma(z)$ is 0.885603194410888 68870 . In all of these values there is the usual likelihood of an error of a unit or so in the terminal digit.

It is interesting to notice that since we are here dealing with an extreme value of the gamma function, the value of $\Gamma(z)$ at the minimum is relatively insensitive to changes in $z$; hence, though it is not possible to determine $z$ to more than eighteen decimals - the extent of Gauss's table of $\psi(z)$ - the limit of accuracy in determining $\log \Gamma(z)$ is not $z$, but is Gauss's table of $\log \Gamma(z+1)$. Hence, our values of $\log \Gamma(z)$ and $\Gamma(z)$ should be correct except for the possibility of errors in the twentieth decimal, as mentioned above. As an illustration of this principle, it might be added that a change in $z$ of one unit in the eleventh decimal place produces a change in log $\Gamma(z)$ of a unit or so in the fifteenth place.

Bureau of Chemistry and Soils ;

W. Edwards Deming.

Bureau of Plant Industry ;

Clatence G. Colcord.

\section{U.S. Department of Agriculture,} Washington, D.C.

\section{Light-Waves as Units of Length}

I wrsh to correct an error that occurs in my article on this subject in NaTURE of March 30 (p. 496). The mean value of the wave-length of red cadmium in normal air from this table should be $6438 \cdot 4691 \times 10^{-10}$ metres, and not $6438.4687 \times 10^{-10}$ metres, which is the mean of the last three determinations.

Originally I considered that only these later results should be used on account of the uncertainty, in the earlier work, regarding the carbon dioxide content of the air. Further consideration at the proof-reading stage led me to include the determination of Benoit, Fabry and Perot, in view of the probability that the actual lengths of the international substandards, No. 26 and $T_{3}$, were known to a greater accuracy with reference to the prototype metre than was the case with the various national standards. The correction was made in the proof, but the revised value for the mean was not inserted. The vacuum wave-length given at the end of the article was derived from this revised value, and therefore needs no alteration.

King's College, W. EWART WILliams.

London, W.C.2. May 2. 